\title{
Virtual drug design: Skp1-Skp2 inhibition targets cancer stem cells
}

\author{
Laura E Pascal $^{1}$ and Zhou Wang ${ }^{1,2,3}$ \\ Asian Journal of Andrology (2013) 15, 717-718; doi:10.1038/aja.2013.115; Published online: 30 September 2013
}

$\mathrm{T}$ he dysregulation of pathways regulating cellular function is a frequent hallmark of cancer and the development of specific pathway inhibitors that alter tumor growth and progression are the focus of multiple recent studies. E3 ubiquitin ligases are a large group of diverse protein enzymes that specifically target proteins for clearance, and their importance to normal cellular function is illustrated in the many diseases associated with their loss of function or inappropriate targeting. S-phase kinase-associated protein $2(\mathrm{Skp} 2)$ is an $\mathrm{F}$ box protein that plays critical roles in cellcycle progression, senescence, metabolism, and acts as an Skp1-Cullin-1-F box (SCF) ubiquitin ligase substrate recognition factor. Overexpression of Skp2 is associated with poor prognosis and metastasis in many cancers and is a well validated drug target. In a recent report, Chan et al. have identified an Skp2 inhibitor that selectively impairs Skp2 E3 ligase activity using an integrated virtual high-throughput drug screening and experimental validation approach. This Skp2 inhibitor restricts cancer stemness and potentiates sensitivity to chemotherapeutic agents in multiple animal tumor models. These findings identify a new novel small molecule that targets the Skp2 and reduces tumor growth by attenuating aerobic glycolysis and inducing cellular senescence.

The F box protein S-phase kinase-associated protein 2 (Skp2) is one of the four subunits of the Skp1-cullin-1-F box (SCF) ubiquitin E3

${ }^{1}$ Department of Urology, University of Pittsburgh Schoo of Medicine, Pittsburgh, PA 15232, USA; ${ }^{2}$ University of Pittsburgh Cancer Institute, University of Pittsburgh School of Medicine, Pittsburgh, PA 15232, USA and ${ }^{3}$ Department of Pharmacology and Chemical Biology, University of Pittsburgh School of Medicine, Pittsburgh, PA 15232, USA

Correspondence: Dr LE Pascal (pascalle@upmc.edu) or Dr Z Wang (wangz2@upmc.edu) ligase complex that can trigger the ubiquitination and degradation of several protein substrates. E3 ubiquitin ligases facilitate the final step of ubiquitin attachment to their specific target protein substrates for proteasome degradation. In the Skp2 SCF complex, Skp2 binds to Skp1, a linker protein, which binds to cullin-1. Skp2 targets several cell-cycle regulator and tumor suppressor proteins, including p27, for ubiquitin-mediated degradation and positively regulates the cell-cycle. Several studies have implicated an oncogenic role for Skp2 and overexpression of Skp2, which leads to increased degradation of p27 and subsequent unchecked cellular proliferation, has been observed in several tumor types. Conversely, Skp2 deficiency triggers p27-dependent cellular senescence and apoptosis. In previous studies, Chan and colleagues ${ }^{1,2}$ demonstrated that the SCF Skp2 complex could also trigger nonproteolytic K63-linked ubiquitination of Akt. The Akt pathway is a critical regulator of cell survival and metabolism. Akt ubiquitination results in enhanced Akt membrane localization and phosphorylation, which increases Akt activation. Akt activation results in aerobic glycolysis, enabling rapid generation of ATP and biomaterials to sustain cancer cell hyperproliferation. Considering that tumor cells express low levels of p27 and activated Akt, specifically disrupting Skp2 E3 ligase function could serve as a promising cancer treatment strategy by reducing both p27 degradation and cell glycolysis, thereby selectively inhibiting cancer cell survival.

Virtual drug screening utilizes informatics approaches to first define hot spot residues and binding pockets and then identify potential inhibitors to block the protein-protein interactions. From these virtual arrangements, predictions can be made as to which molecules will bind most effectively, while those with low binding affinities are excluded. This method can efficiently sift through hundreds of thousands of candidate molecules to identify a workable number of potential compounds that target the protein of interest. These potential inhibitors will then be taken to the wet lab for validation. Recently the identification of compounds that could specifically target and inhibit E3 ligase activity has generated much enthusiasm. Wu et al. ${ }^{3}$ previously utilized an in silico structure-based virtual ligand screening strategy to identify compounds that could specifically inhibit the Skp2-mediated degradation of p27 by disrupting phosphor-p27 and Skp2-Cks1 interaction. These small molecules were shown to interrupt Skp2p27 interaction, resulting in reduced ubiquitilyation and increased stability of p27. Furthermore, the compounds were cable of inhibiting p27- and Skp2-dependent cellcycle progression in several cancer cell lines, resulting in decreased proliferation. However, these compounds were highly specific to the Skp2-p27 interface and did not disrupt formation of the Skp1-Skp2 complex, suggesting that the degradation of other SCF Skp2 ligase targets would remain unaffected.

In a recent paper in Cell, Chan and colleagues $^{4}$ utilized a high throughput virtual screening approach to identify residue 'hot spots' along the Skp2-Skp1 interaction surface. These hot spots are residues along the Skp2-Skp1 interaction interface that contribute more significantly to their binding. The authors identified 19 such potential residues and grouped them according to proximity to the $\mathrm{N}$-terminus or the $\mathrm{C}$-terminus of Skp2. Inhibitors were screened in silico using their previously developed high-performance computing-based molecular docking scheme, termed HiPCDock, ${ }^{5}$ for chemical compounds predicted to bind at either or both sites that might block the binding of Skp1 to Skp2. The candidate inhibitors were screened for their ability to prevent Skp2 and Skp1 interaction 
in vitro with a glutathione $S$-transferase pulldown assay. Biochemical analyses in prostate cancer cell lines verified that candidate compound \#25 could stabilize expression of Skp2 canonical substrates p27 and p21, as well as inhibit the activation of Akt. Further experiments in cell lines indicated that compound \#25 was specific to Skp2 E3 ligase activity, but not other $\mathrm{F}$ box proteins.

Cancer stem cells (CSCs) or cancer initiating cells express stem cell markers and are capable of self-renewal and asymmetric cell division. Several studies have postulated that CSCs can be the cells of origin for cancers and are responsible for tumor progression and metastasis. Additionally, tumor relapse is thought to be caused by the survival of small CSC populations that resist conventional treatment. Chan and colleagues showed that Skp2 inactivation reduced the CSC population in prostate cancer cell lines and inhibited the ability of prostate CSCs to form prostate spheres, suggesting that Skp2 inhibition suppressed the self-renewal capacity of CSCs. In xenograft experiments, the Skp2 inhibitor compound \#25 enhanced prostate tumor response to doxorubicin or cyclophosphamide. Furthermore, they demonstrated that treatment of established lung or prostate tumor xenografts with compound \#25 induced an upregulation of p27, p21 and apoptosis and a decrease in cellular proliferation. Glycolysis regulators Akt and Glut1 were also downregulated, suggesting that Skp2 inactivation could attenuate aerobic glycolysis in xenograft tumors.

The authors identified a diverse set of compounds using an in silico high-throughput virtual screening method to quickly sift through a large chemical database containing 120000 commercially available compounds. Among them, 25 were selected to be validated in the wet lab for their specificity in preventing Skp2Skp1 interaction, inhibiting Skp2 SCF E3 ligase activity toward ubiquitination of p27 and Akt, and repression of tumor xenograft growth. Chan and colleagues also revealed a critical role for Skp2 in governing CSC properties, including their capacity for self-renewal. Pharmacological inactivation of Skp2 in tumors could confer sensitivity to chemotherapy and reduce tumor progression by disrupting the ability of CSCs to survive and resist treatment. Translation of Skp2 inhibition as a cancer treatment strategy to the clinic will require the identification of additional Skp2 target proteins disrupted by these small molecules, their effect on untransformed cells and the potential for parallel degradation pathways to compensate for the loss of Skp2 function. This current study illustrates the power of using virtual molecular structurebased modeling, high-throughput in silico screening and experimental validation to develop highly specific targeting and inhibition of E3-substrate interactions. This study illustrates a promising new therapeutic strategy targeting Skp2 E3 ligase that could potentially be used to kill the elusive cancer stem cell.

1 Chan CH, Li CF, Yang WL, Gao Y, Lee SW et al. The Skp2-SCF E3 ligase regulates Akt ubiquitination, glycolysis, herceptin sensitivity, and tumorigenesis. Cell 2012; 149: 1098-111.

2 Yang WL, Wang J, Chan CH, Lee SW, Campos AD et al. The E3 ligase TRAF6 regulates Akt ubiquitination and activation. Science 2009; 325: 1134-8.

3 Wu L, Grigoryan AV, Li Y, Hao B, Pagano M et al. Specific small molecule inhibitors of Skp2-mediated p27 degradation. Chem Biol 2012; 19: 1515-24.

4 Chan CH, Morrow JK, Li CF, Gao Y, Jin G et al. Pharmacological inactivation of Skp2 SCF ubiquitin ligase restricts cancer stem cell traits and cancer progression. Cell 2013; 154: 556-68.

5 Zhang S, Du-Cuny L. Development and evaluation of a new statistical model for structure-based highthroughput virtual screening. Int J Bioinform Res App/ 2009; 5: 269-79. 